\title{
UMA ANÁLISE DA INOVAÇÃO NA AGROINDÚSTRIA NO BRASIL ${ }^{1}$
}

\author{
Júnia Cristina P. R. da Conceição ${ }^{2}$ \\ Mansueto Almeida ${ }^{3}$
}

Resumo - Este trabalho objetivou identificar as características das empresas do setor de alimentos e bebidas, no que se refere às inovações tecnológicas. Do ponto de vista dos condicionantes microeconômicos à inovação, os resultados indicam que o tamanho da firma, a escolaridade do trabalhador e os esforços em $\mathrm{P} \& \mathrm{D}$ apresentam impacto positivo na probabilidade de a firma inovar. Além disso, os resultados encontrados apontam para o fato de que a inovação feita pelas firmas é condição necessária para o atendimento do mercado consumidor interno, seja no segmento do mercado mais exigente em qualidade, seja no de menor poder aquisitivo. Assim, o consumidor interno acaba por desempenhar papel fundamental na indução de inovações tecnológicas neste setor, pelo menos no período analisado de 1998 a 2000.

Palavras-chave: agroindústria, inovação tecnológica, Brasil.

\section{Introdução}

Com a abertura do mercado nacional e estabilização da moeda, as empresas brasileiras passaram a utilizar diversas estratégias tanto para manter o número de clientes, como para ampliá-lo. Fundamentadas em um novo referencial de competição, as empresas buscaram estabelecer, na indústria nacional, capacidade competitiva para atuar positivamente junto ao consumidor.

Recebido em 26/08/2005. Aceito em 05/10/2005.

Pesquisadora do IPEA.

3 Pesquisador do IPEA. 
No caso específico do setor agroindustrial, diversos estudiosos têm reconhecido uma ampla interface no processo de inovação tecnológica com outros setores industriais. A indústria de alimentos estimula inovações em toda a cadeia agroalimentar, como nos produtores de matéria-prima (agricultura), no setor de embalagens, na distribuição atacadista e varejista, e em indústrias de bens de capital.

Dessa forma, a identificação de variáveis que potencialmente expliquem a inovação tecnológica em empresas alimentares será útil para o entendimento do processo inovador dentro desta indústria e também em indústrias com ela relacionadas.

Atualmente, as empresas do setor de alimentos no Brasil encontram-se numa posição em que os recursos tecnológicos passam a ser fundamentais para que elas possam enfrentar os desafios da competição nacional e internacional. Isso porquê a abertura comercial não só trouxe novas oportunidades de mercado no exterior, mas também facilitou a presença de competidores estrangeiros no país.

É exatamente por isso que a inovação tecnológica tem sido identificada como uma das principais fontes de vantagem competitiva para as empresas. Nesse sentido, diversos trabalhos têm sido desenvolvidos para identificar os fatores que estimulam a inovação tecnológica nas firmas, e é este também o objetivo principal deste trabalho.

Pelo menos três abordagens analíticas no campo da economia têm se destacado no estudo de processos de inovação ${ }^{4}$. A primeira se refere ao debate entre tamanho de empresa e estrutura (concentração) de mercado; a segunda, aos estudos que analisam a força primária da inovação, oferta ou demanda (technology-push versus demand-pull); a terceira, aos estudos no âmbito da teoria evolucionista (evolutionary economics) e da teoria baseada nos recursos das empresas (the resource-based theory of the firm), os quais analisam os determinantes dos padrões de inovação na indústria e nas empresas. Nesse caso, na investigação dos

${ }^{4}$ O trabalho de Cabral (2001) apresenta uma discussão destas abordagens. 
determinantes da inovação levaram-se em consideração tanto a intensidade e a direção (produto ou processo) da inovação, como a origem (onde a inovação foi gerada), a complexidade (radical ou incremental) e a novidade (nova ou imitação).

As críticas em relação às duas primeiras abordagens são múltiplas. A abordagem technology-push é criticada em virtude, essencialmente, da sua dificuldade em levar em conta o fato óbvio de que fatores econômicos desempenham papel importante na determinação da direção da mudança tecnológica. Quanto à abordagem demand-pull, ela falha, principalmente, por causa de sua dificuldade em explicar quando desenvolvimentos tecnológicos específicos ocorrem e porque a capacidade de invenção muda com o tempo, sem qualquer relação direta com a mudança das condições de mercado.

Tendo em vista a discussão anterior, neste trabalho procurar-se-á identificar os determinantes microeconômicos da firma que impulsionam a adoção da inovação tecnológica e também o papel desempenhado pelo consumidor (interno e externo) na adoção de inovações tecnológicas. $\mathrm{Na}$ realidade, pressupõe-se que, no caso da agroindústria, tanto as características das firmas quanto a sinalização dada pelo mercado são importantes para determinar o tipo e o grau das inovações tecnológicas e exercem influência positiva no desencadeamento do processo inovativo.

Com base nesse referencial, será possível utilizar essas informações para avaliar, ainda que de forma exploratória, a associação existente entre algumas variáveis e a capacidade inovativa das firmas integrantes da indústria de alimentos. É exatamente nesse contexto que se situa o presente artigo, que é organizado em três seções, além desta introdução. Na primeira seção, apresenta-se revisão de literatura, que fornece subsídios para a discussão e também para a construção de hipóteses que serão posteriormente testadas; na segunda, uma análise econométrica, pela qual se procure relacionar algumas variáveis de tamanho, origem do capital, escolaridade dos trabalhadores e papel desempenhado pelos consumidores na adoção de tecnologia; finalmente, na última, são apresentadas as principais conclusões do trabalho e sugestões de políticas 
públicas para a Política Industrial Tecnológica e de Comércio Exterior (PITCE), no que se refere ao setor agroindustrial.

\section{Revisão de literatura: o papel do consumidor no desencadeamento da inovação das firmas do setor de ali- mentos}

A inovação e o sucesso da inovação dependem, em larga escala, do comportamento do consumidor. Traill (1997) argumentou que mudanças no padrão de consumo determinam, freqüentemente, a natureza da inovação que ocorre na indústria.

Gracia e Albisu (1997) chamaram atenção para o fato de que existem fatores relacionados com comportamento do consumidor, tais como nível de renda, características demográficas e culturais, que também afetam as decisões de consumo.

Becattini (1994) enfatizou que, no caso específico da agroindústria, aspectos relacionados com qualidade e segurança do produto (food safety) passaram a ser importantes na decisão do consumo. Os consumidores, principalmente os de faixa de renda mais elevada, passaram a demandar produtos alimentícios com mais atributos (congelados, pronto para consumir, naturais e lights e diets). Esse comportamento do consumidor teve impacto positivo no processo inovativo por parte das empresas, que tiveram que se adaptar às novas exigências por parte dos consumidores.

No caso específico do Brasil, a condição de estabilização econômica do país, a partir de 1995, permitiu ao consumidor acesso a novos produtos e condições de compra. Silveira e Lepsch (1997) observaram que, no acirramento da competição entre as firmas, o principal componente era o consumidor, que ficou mais exigente. Assim, o comportamento do consumidor, influenciado pela nova condição econômica, pelo acesso à informação e pelo contato com novos produtos provenientes do mercado externo, estabeleceu uma intensificação da competição, o que exigiu novas estratégias do setor no que se refere à inovação. 
Wedekin e Neves (1995) chamaram atenção para o forte impacto das alterações nos hábitos, gostos e preferências do consumidor, bem como na mudança em seu estilo de vida, o que produziu novas demandas no setor agroindustrial. Segundo esses autores, surgiram novos paradigmas de consumo, quais sejam, diferenciação por meio de aspectos qualitativos; interação entre consumo de alimentos e meio ambiente; e valorização da saúde. Os efeitos desse fenômeno sobre as firmas do setor são intensa competição por market share; menor ciclo de vida dos produtos; profusão de novos lançamentos; modificações nas tecnologias de produção, transporte e embalagens; novos modos de compra e consumo; novos modos de comunicação com o consumidor final; e segurança do consumidor.

Nesse contexto, a agroindústria passou a empregar estratégias que possibilitassem a implementação de competência técnica e operacional que envolvia produto e processo, para enfrentar a competição dentro do novo mercado global e assegurar sua capacidade de sobrevivência e expansão.

Nesse novo cenário, aspectos como diferenciação de produtos por meio de aspectos qualitativos passam a ganhar nova dimensão. Como argumentaram Toledo et al. (1997), as empresas do setor procuram atender aos desejos dos consumidores. Dib (1997) observou que as modificações no comportamento do consumidor estabelecem novos hábitos de compra, além da preocupação maior com a saúde, com segurança dos alimentos ingeridos e com suas características nutricionais. Segundo ele, as mudanças seguem o ritmo da introdução e adoção de novas tecnologias e descobertas nas áreas da fisiologia, nutrição e bioquímica. O sistema alimentar passa a contar com novos paradigmas de consumo - a diferenciação por meio de aspectos qualitativos; a diversidade da demanda; e a valorização da saúde, cujos efeitos são intensa competição por market share, menor ciclo de vida dos produtos e novos lançamentos. 
Peppers (1997) salientou que o comportamento do consumidor evoluiu para uma situação não mais de busca de opções, mas de encontrar no mercado exatamente aquilo que desejam, e a tecnologia passa a ser a ferramenta necessária para o atendimento dessas novas exigências. Como salientou Barzel (1982), conceitos sobre segurança dos produtos alimentares passaram a fazer parte do cotidiano das pessoas.

\section{Principais fatores determinantes da adoção de inovação no setor de alimentos}

$\mathrm{Na}$ tentativa de estabelecer relações de causalidade entre algumas variáveis selecionadas e adoção de práticas inovativas, foi testado um modelo PROBIT ${ }^{5}$, cuja escolha se deveu ao fato de a variável dependente ser binária (faz ou não inovação). As variáveis explicativas foram selecionadas com base no que for discutido na literatura sobre inovação na indústria de alimentos (veja, por exemplo, Cabral, 2001, e Gallizzi e Venturini, 1996). Ademais, pretende-se também testar formalmente a hipótese de que, além das características microeconômicas das firmas, o consumidor, como ressaltado anteriormente, tem influência positiva no desencadeamento das inovações.

Foram utilizados três bancos de dados ${ }^{6}$ : - Pintec/IBGE, PIA/IBGE, Secex/ MDIC e RAIS/TEM. A análise feita foi cross-section, tendo em vista que os dados da PINTEC, que se referem ao ano 2000.

As variáveis independentes utilizadas nos modelos foram ${ }^{7}$ :

- Valor da Transformação Industrial /Pessoal ocupado: Esta variável foi utilizada como proxy de produtividade da firma;

\footnotetext{
Foi utilizado o software SAS na estimação do modelo Probit.

6 Na realidade, o Instituto de Pesquisa Econômica Aplicada (IPEA), especificamente a Diretoria de Estudos Setoriais (DISET), organizou uma base de dados com informações por firmas. De Negri (2003) explicitou, com detalhes, a construção deste banco de dados.

7 As variáveis explicativas foram utilizadas nos logaritmos.
} 
- Gastos com Propaganda/Faturamento: Valor das despesas com propaganda pagas ou creditadas a terceiros em relação ao faturamento;

- Escolaridade: É o tempo médio de estudo do trabalhador;

- Inovativo: Esta variável foi obtida pela agregação das variáveis valor do dispêndio referente $\mathrm{a} \& \mathrm{D}$, valor do dispêndio relativo à aquisição externa de $\mathrm{P} \& \mathrm{D}$, valor do dispêndio relacionado com aquisição de outros conhecimentos externos, valor do dispêndio com treinamento, valor referente à introdução das inovações tecnológicas no mercado e valor do dispêndio referente ao projeto industrial e outras preparações técnicas para produção e distribuição. É a soma dos esforços de P\&D efetuados pelas empresas em relação à receita líquida de vendas.As variáveis que compõem esta variável são definidas, na PINTEC, por atividades representativas dos esforços da empresa voltados para a melhoria do seu acervo tecnológico;

- Pessoal Ocupado: É o número de pessoal ocupado por firma. Foi utilizado como proxy de tamanho;

- Dummies para capital: Procurou-se, por esta variável, verificar se a origem do capital tinha influência sobre a probabilidade de adoção de tecnologia. O capital controlador foi classificado em nacional ou estrangeiro.

- Dummies para Subsetores da Indústria de alimentos: Definida de acordo com a CNAE 3, com o intuito de controlar as especificidades de cada subsetor, no que diz respeito aos esforços de P\&D.

- Exportação: Foi definida como o valor total das exportações realizadas pela firma em relação ao seu faturamento

- Dummy Consumidor: Esta variável foi classificada como igual a 1, no caso de a empresa atribuir alta importância de consumidores como fonte de informação para desenvolvimento de produtos/processos 
tecnologicamente novos ou substancialmente aprimorados. Tenta capturar a importância do consumidor como estímulo à inovação. Será igual a 1, quando a importância for classificada como alta e zero, caso contrário.

- Dummy Localização do Consumidor: Esta variável identifica a localização de clientes ou consumidores, utilizada como fonte de informação para a empresa ter desenvolvido-produtos e, ou, processos tecnologicamente novos ou aprimorados. Será igual a 1, quando a resposta for o Brasil e zero, quando a resposta for o exterior. Permite inferir sobre qual demanda, interna ou externa, é mais importante na determinação da inovação tecnológica, no caso do setor de produtos alimentares.

- Dummy Cooperação: Investiga se a empresa esteve envolvida em arranjos cooperativos com outras organizações para o desenvolvimento das inovações. Será igual a um, se a empresa estiver envolvida e zero, caso contrário.

\section{Resultados}

Os resultados do modelo estimado, em que a variável dependente era se a firma faz ou não inovação, independente do tipo (produto ou processo) e independente de ser para empresa ou para o mercado, estão expressos na Tabela 1. A análise dos resultados pode ser feita diretamente a partir do efeito marginal, que fornece o impacto de cada variável na probabilidade de a firma ser inovadora.

Os resultados indicam que as variáveis VTI/Pessoal Ocupado, gastos com propaganda/faturamento, escolaridade, esforços de $\mathrm{P} \& \mathrm{D}$ e pessoal ocupado tiveram impacto positivo e estatisticamente significativo na probabilidade de a firma inovar. Destes, o que teve maior impacto na probabilidade de a firma inovar foi o esforço em P\&D. Os resultados mostram que aumento de $10 \%$ nos esforços de $\mathrm{P} \& \mathrm{D}$ ocasionaria aumento de $10 \%$ na probabilidade de a firma adotar inovações, seja de produto seja de processo. 
Outro fato importante de se registrar é a importância do consumidor no desencadeamento da inovação. Mais do que isso, a variável dummy, para o consumidor brasileiro, foi positiva, o que indica que, quando o consumidor está no Brasil, há aumento na probabilidade de a firma inovar diante do consumidor externo. A variável dummy para cooperação também foi significativa, o que demonstra a importância da cooperação para o desenvolvimento de atividades inovativas na indústria de alimentos. Das variáveis explicativas utilizadas, as dummies para capital e as exportações não foram significativas, ou seja, não interferiram na probabilidade de a firma inovar.

A relação positiva entre tamanho e inovação, presente na literatura Kamien e Schwartz (1982) e Cohen e Levin (1989), também foi comprovada neste estudo. A variável inovativo foi positiva e significativa, o que demonstra sua importância para a inovação. Alguns estudos sugerem que aumento nos gastos com esforço inovativo implica número maior de inovação de produto (Galizzi e Venturini, 1996). Os resultados encontrados neste trabalho reforçam essa idéia. 
Tabela 1 - Modelo I (variável dependente: firmas inovadoras)

\begin{tabular}{lcc}
\hline \multicolumn{1}{c}{ Variáveis } & Parâmetros & Efeito Marginal \\
\hline Intercepto & $-0,2515^{\text {ns }}$ & $-0,0956$ \\
Vti_pol & $0,1475^{*}$ & 0,0561 \\
Gastos Propaganda/Faturamento & $0,0997^{* * *}$ & 0,0379 \\
Propaganda/Faturamento2 & $0,0048^{* * *}$ & 0,0018 \\
Escolaridade & $0,3891^{*}$ & 0,1480 \\
Pessoal Ocupado & $0,3312^{*}$ & 0,1259 \\
Inovativo & $0,2895^{*}$ & 0,1101 \\
Origem do Capital & $-0,091^{\text {ns }}$ & $-0,0346$ \\
Exportações & $0,0429^{\text {ns }}$ & 0,0163 \\
Dummy Consumidor & $0,4746^{*}$ & 0,1805 \\
Consumidor (Interno, Externo) & $1,5609^{*}$ & 0,5937 \\
Cooperação & $0,8636^{*}$ & 0,3284 \\
cnae 151 & $0,1831^{\text {ns }}$ & 0,0696 \\
cnae 152 & $1,1^{*}$ & 0,4185 \\
cnae 153 & $0,0216^{\text {ns }}$ & 0,0082 \\
cnae 154 & $0,0593^{\text {ns }}$ & 0,0225 \\
cnae 155 & $0,2419^{* * *}$ & 0,092 \\
cnae 156 & $-0,0455^{\text {ns }}$ & $-0,0173$ \\
cnae 157 & $-0,2871^{* * *}$ & $-0,10921$ \\
cnae 158 & $0,1686^{* * *}$ & 0,0641 \\
\hline Log Likelihood Function LL $(\beta)$ & $-422,81$ & \\
Restr.Log Likelihood Function LL(0) & $-6173,2$ & \\
Pseudo R ${ }^{2}$ & 0,789 & \\
\hline Fon & Pintec/IBGE, PIA/IBGE, Secx//DD & \\
\hline
\end{tabular}

Fonte: Pintec/IBGE, PIA/IBGE, Secex/MDIC e RAIS/TEM.

Legenda: ns: não-significativo.

$* * *$ significativo a $1 \%$.

$* *$ significativo a $5 \%$.

* significativo a $10 \%$.

Os resultados indicam que as variáveis mais importantes para implementação de atividades inovativas de processo na indústria de alimentos são o tamanho (medido pelo pessoal ocupado), os gastos com esforços inovativos e o consumidor, principalmente o nacional. A dummy

\footnotetext{
8 Pseudo R2, calculado segundo Greene, W. H. Econometric Analysis.
} 
para capital estrangeiro foi positiva, com efeito marginal de 0,308 . A cooperação também aparece como variável importante para desencadear o processo inovativo. Foram constatadas diferenças na probabilidade de adoção de inovações entre as categorias $\mathrm{A}, \mathrm{B}$ e C, por meio das variáveis dummies.

Os setores mais inovativos são 152 (processamento, preservação e produção de conservas de frutas legumes), 154 (laticínios), 155 (moagem, fabricação de produtos amiláceos e de rações balanceadas para animais), 156 (fabricação e refino de açúcar) e 158 (fabricação de outros produtos alimentícios). As variáveis dummies nesses setores foram positivas e estatisticamente significativas. Um ponto que merece ser destacado é que os efeitos marginais foram significativamente inferiores aos do primeiro modelo. Isso era de esperar, já que este segundo modelo trata apenas das firmas que fazem inovação de processo para mercado.

\section{Síntese dos principais resultados}

A estimação do modelo econométrico também apresentou resultados interessantes. Os números indicam que os índices de inovação entre as grandes e médias empresas foram maiores do que os das duas pequenas. Esse resultado está de acordo com o postulado na teoria sobre inovação tecnológica, segundo o qual as inovações seriam mais lucrativas em grandes firmas, tendo em vista a possibilidade de redução nos custos unitários de produção ou, em outras palavras, nas economias de escala. Esse resultado está de acordo tanto com o de Cabral (2000) quanto com o de Gallizi e Venturini (1996).

Outra variável que se mostrou importante foi gasto com propaganda. A propaganda é uma função de marketing considerada muito importante para as empresas alimentícias, particularmente quando associadas a inovações (especialmente em produto). Connor e Schiek (1997) afirmaram que os esforços de propaganda desempenham papel-chave nos objetivos das empresas alimentícias na indução dos clientes ao teste 
de novos produtos, atração da atenção deles para novas marcas e manutenção da lealdade às marcas estabelecidas no mercado. Os resultados dos modelos estimados confirmam a importância da propaganda na probabilidade de a firma inovar.

A educação tem grande importância para que as empresas do setor de alimentação desenvolvam atividades inovativas, seja de processo, seja de produto. Gastos com Pesquisa e Desenvolvimento também apresentam efeito positivo e estatisticamente significativos sobre a probabilidade de a firma adotar inovações.

Outra variável importante foi a cooperação. Há proposições teóricas de que o esforço de $\mathrm{P} \& \mathrm{D}$ das empresas alimentícias se refere, em boa escala, a pesquisas realizadas por outras instituições ou em parceria com elas. A idéia básica é de que não apenas para a indústria de alimentos, um modelo de pesquisa cooperativo é mais eficiente no desenho e desenvolvimento de novos produtos e processos (ver, por exemplo, Connor e Schiek, 1997 e Cabral, 2000).

Um fato que chamou atenção foi a não-significância da variável exportação sobre a probabilidade de a firma realizar inovações. Essa variável não foi significativa em nenhum dos modelos, apesar da expectativa de que ela fosse. Pode-se argumentar que as empresas líderes desviaram sua atenção para o mercado interno, que ficou aquecido depois do programa de estabilização macroeconômica de 1994. Esse resultado é confirmado, inclusive, pela significância da variável que capta a importância do mercado doméstico no desencadeamento de inovações tecnológicas. De fato, no caso das indústrias alimentares, o mercado consumidor interno é importante para o aumento da probabilidade de a firma inovar. Esse resultado é importante e precisa ser lembrado na definição de instrumentos de política industrial para o setor.

A análise das variáveis dummies para os setores foi significativa para alguns deles, a exemplo do 152 (processamento, preservação e produção de conservas de frutas, legumes e outros), 154 (laticínios), 155 (moagem, fabricação de produtos amiláceos e de rações balanceadas para animais) 
158 (fabricação de outros produtos alimentícios). Como as dummies tiveram sinal positivo, pode-se inferir que, nesses setores, a iniciativa para inovar, controlada as demais variáveis, foi maior.

\section{Conclusão}

No Brasil, as exigências de competitividade no setor de agroalimentar estão se tornando cada vez mais sofisticadas. Por isso, os agentes do sistema agroalimentar brasileiro têm, necessariamente, a obrigação de determinar seu lugar na competição tecnológica e industrial.

Os resultados desta pesquisa identificam alguns fatores importantes que podem contribuir para o delineamento de instrumentos que possam acelerar o desencadeamento de inovações no setor agroindustrial. Um ponto a ser ressaltado é o fato de que, além do aumento, da diversificação e da funcionalidade dos produtos e processos de produção, o mercado alimentar atual exige também qualidade.

A melhoria na qualidade dos produtos não é condição necessária somente para a exportação, mas também para o atendimento do mercado consumidor interno, que tem se mostrado mais exigente. Assim, desempenha papel fundamental na indução de inovações tecnológicas.

Como observou Cribb (2004), atualmente, há necessidade de reestruturação do sistema agroalimentar brasileiro, que precisa ser concebida não apenas em termos quantitativos, mas também qualitativos, para acompanhar a evolução do mercado alimentar, pois estão ocorrendo mudanças extremamente importantes no consumo agroalimentar.

Confirmou-se a hipótese formulada de que neste setor, pelo menos por enquanto, o mercado consumidor interno tem mais relevância para desencadear processos inovativos do que o setor externo. 
Este trabalho demonstrou que há, ainda, grande espaço para que as firmas do setor agroalimentar melhorem seu desempenho, pois, como visto, o percentual de adoção de inovações é ainda pequeno.

\section{Classificação CNAE}

151 - Abate e preparação de produtos de carne e de pescado

152 - Processamento, preservação e produção de conservas de frutas, legumes e outros

153 - Produção de óleos e gorduras vegetais e animais

154 - Laticínios

155 - Moagem, fabricação de produtos amiláceos e de rações balanceadas para animais

156 - Fabricação e refino de açúcar

157 - Torrefação e moagem de café

158 - Fabricação de outros produtos alimentícios

159 - Fabricação de bebidas

\section{Referências bibliográficas}

BARZEL, Y. Measurement Cost and Organization of Markets. Journal of Law and Economics, 25 April, 1982.

BECCATINI, G. O distrito marshalliano. In Benko,G; Lipietz, A (org.) As regiões Ganhadoras. Oeiras: Celta Editora.1994.

CABRAL, J.E.O. Determinants of firm's likelihood to innovate and intensity of innovation in the Brazilian food industry. The Journal on Chain and Network Science 01, 33-48, 2001.

COHEN, W.M. and LEVIN, R.C. Empirical Studies of Innovation and Market Structure. In Schmalensee, R. and Willig, R.D. Handbook of Industrial Organization. Vol II Amsterdan, 1989. 
DE NEGRI, J.A. \& Salerno, M.S. (orgs) Inovações, Padrões Tecnológicos e Desempenho das Firmas Industriais Brasileiras.Brasília: IPEA, 2005.

DIB, J. A. Efficient consumer response (ECR): uma estratégia para o varejo de bens de consumo de massa. Revista de Administração, São Paulo, v.2, n.12, p.14-22, abr/jun, 1997.

GRACIA, A.\& ALBISU, L.M. Consumption patterns in Western Europe. In: Globaliztion of the food industry:policy implications. Loader, Henson and Traill (eds) University of Reading.1997.

GREENE,W. H. Econometric Analysis. Prentice Hall, 1997.

GALIZZI, G and VENTURINI, L. Economics of Innovation. The case of Food Industry. Heidelbergh, 1996.

KAMIEN, M. I. \& SCHWARTZ, N. Market Structure and Innovation. Cambridge, 1982.

PINTEC/IBGE.

PEPPERS, D. ROGERS, M. Enterprise one to one: Tools for competting in interecative age. New York, 1997.

TRAILL, B. Structural Changes in European food industry: consequences of innovation. In Traill and Grunert (eds). Product and process innovation in the food industry. Chapman and Hall, London.

TOLEDO, L. et alli. Marketing estratégico e varejo: o caso europeu. Revista de Administração, São Paulo, v.32, n.1, p.47-57, abr./jun.1997.

SILVEIRA, J. A G. \& LEPSCH, S.L. Alterações Recentes na Economia do Setor Supermercadista Brasileiro. Revista de Administração da USP. São Paulo, v.32,n2 abr./jun., 1997. 
WEDEKIN, I; Neves, M.F. Sistema de Distribuição de Alimentos: Impacto das novas tecnologias. Revista de Administração de Empresas. São Paulo, v.30, n.4, p 15-18, out./dez. 1995.

Abstract - This paper seeks to identify the major factors behind the innovation process of firms in the food and beverage sector. On the microeconomic incentives to innovate, our results show that larger firms, higher schooling and higher R\&D expenditures increase the probability that firms will innovate. In addition, our results show that innovation is essential for food and beverage firms to meet the domestic demand, including the upper segment of the consumer market and also the bottom one. At last, our results indicate that the domestic consumer was a key factor behind food and beverage firms' incentives to innovate in the 1998-2000 period.

Key-words: agro industry, innovation, Brazil. 